\title{
TAMANHO E FORMA DE PARCELAS EXPERIMENTAIS PARA Eremanthus erythropappus
}

\author{
Gabriel Marcos Vieira Oliveira ${ }^{1}$, José Márcio de Mello² $^{2}$, Renato Ribeiro de Lima ${ }^{3}$, \\ José Roberto Soares Scolforo ${ }^{4}$, Antônio Donizette de Oliveira ${ }^{5}$
}

(recebido: 23 de junho de 2010; aceito: 28 de abril de 2011)

RESUMO: O tamanho adequado e a forma das parcelas experimentais para candeia é uma alternativa para garantir interpretações corretas e conclusões bem fundamentadas sobre o cultivo dessa espécie. Assim, neste trabalho, objetivou-se definir o tamanho ótimo, tamanhos convenientes e forma adequada de parcelas experimentais para Eremanthus erythropappus, para avaliação das principais características dendrométricas em plantios experimentais. Para isso, foi utilizado um ensaio de uniformidade contendo 400 plantas, as quais tiveram a circunferência, a altura e o volume determinados. Foram simuladas 31 combinações de tamanho/forma de parcelas experimentais. Para a determinação do tamanho ótimo, foram utilizados três métodos de curvatura e um método baseado no modelo linear segmentado com platô. A forma adequada foi determinada a partir do critério de informação relativa e coeficiente de variação. O tamanho conveniente foi estimado pelo de método Hatheway. Os resultados e as conclusões obtidas foram as de que o tamanho de parcelas experimentais para candeia, de modo a permitir análise das principais características dendrométricas, deve ser de 60 plantas úteis, sendo a conformação retangular $2 \times 30$ (linhas $\times$ plantas) a forma mais adequada. O tamanho conveniente é muito flexível e permite adequação do tamanho da parcela experimental com as condições, características e limitações do experimento.

Palavras-chave: Candeia, ensaio de uniformidade, máxima curvatura, modelo linear segmentado com platô, método de Hateway.

\section{SIZE AND SHAPE OF EXPERIMENTAL PLOTS FOR Eremanthus erythropappus}

\begin{abstract}
Defining a suitable size and shape for experimental plots of candeia is an alternative for ensuring correct interpretations and consistent conclusions about the cultivation of this species. The objective of this work was to define optimal size, convenient sizes and suitable shape for experimental plots of Eremanthus erythropappus, for evaluation of main dendrometric characteristics in experimental plantations. To attain that, a uniformity trial was used containing 400 plants, all of which had circumference, height and volume measured. 31 combinations of size/shape were simulated for experimental plots. For determination of optimal size, three curvature methods and one method based on the segmented linear plateau model were used. For determination of suitable shape, the criterion of relative information and coefficient of variation was used. Convenient size was estimated by the Hatheway method. Results led to the conclusion that the size of experimental plots of candeia capable of allowing analysis of main dendrometric characteristics should be 60 useful plants, the most suitable shape being a rectangular configuration of $2 \times 30$ (rows $\times$ plants). Convenient size is a very flexible parameter and allows adaptations depending on the conditions, characteristics and limitations of the experiment in question.
\end{abstract}

Key words: Candeia, uniformity trial, maximum curvature, segmented linear plateau model, Hatheway method.

\section{INTRODUÇÃO}

Durante décadas, as espécies do gênero Eremanthus sp. conhecidas popularmente como candeia, são utilizadas para a produção de moirões de cercas ou para extração do seu óleo essencial para obtenção do componente alfabisabolol. Esses produtos alcançam preços altos no mercado (OLIVEIRA et al., 2009; SCOLFORO et al., 2008).
A obtenção desses produtos é advinda da exploração predatória de candeais nativos e, na melhor das hipóteses, amparadas por planos de manejo. Porém, o acesso aos candeais nativos é cada vez mais restrito. A alternativa que as empresas estão adotando é o plantio comercial de candeais, porém pouco se sabe sobre o seu cultivo e todas as informações, até então, vieram dos candeais nativos ou de experimentos em nível de casa de vegetação ou viveiro.

\footnotetext{
${ }^{1}$ Engenheiro Florestal, Doutorando em Engenharia Florestal - Departamento de Ciências Florestais - Universidade Federal de Lavras - Cx. P. 3037 37200-000 - Lavras, MG - gabrielmvo@gmail.com

${ }^{2}$ Engenheiro Florestal, Professor Doutor em Recursos Florestais - Departamento de Ciências Florestais - Universidade Federal de Lavras - Cx. P. 3037 37200-000 - Lavras, MG - josemarcio@dcf.ufla.br

${ }^{3}$ Zootecnista, Professor Dr. em Estatística e Experimentação Agronômica - Departamento de Ciências Exatas - Universidade Federal de Lavras Cx. P. 3037 -37200-000 - Lavras, MG - rrlima@dex.ufla.br

${ }^{4}$ Engenheiro Florestal, Professor Doutor em Engenharia Florestal - Departamento de Ciências Florestais - Universidade Federal de Lavras - Cx. P. 3037 37200-000 - Lavras, MG - scolforo@dcf.ufla.br

${ }^{5}$ Engenheiro Florestal, Professor Dr. em Ciência Florestal - Departamento de Ciências Florestais - Universidade Federal de Lavras - Cx. P. 3037 37200-000 - Lavras, MG - donizete@dcf.ufla.br
}

Cerne, Lavras, v. 17, n. 3, p. 327-338, jul./set. 2011 
Existe pouca experiência com experimentação em nível de campo com a candeia, sendo que, alguns dos poucos exemplos, podem ser vistos em Scolforo et al. (2008) e Silva (2009). É de se esperar que experimentos como esses se intensifiquem, tendo em vista o potencial econômico dessa espécie.

De acordo com Donato et al. (2008), em experimentos convencionais, procura-se detectar diferenças significativas entre tratamentos, o que é afetado pelo tamanho e a forma da parcela experimental. Segundo Muniz et al. (2009), a escolha criteriosa desses fatores influi na redução do efeito da variabilidade experimental sobre os resultados experimentais, melhorando a precisão, reduzindo o erro experimental, aumentando as chances de se detectar diferenças entre os tratamentos.

O tamanho ótimo da parcela é, muitas vezes, recomendado por meio de estudos empíricos ou, segundo Silva et al. (2003), são definidos de acordo com as necessidades e a experiência do pesquisador. Mas, idealmente deveriam ser baseados em métodos próprios, que buscam estimar quais os tamanhos mais convenientes para reduzir o erro experimental e, consequentemente, obter alta precisão com menor área possível (STORCK et al., 2006; ZANON; STORK, 2000).

A maioria dos vários métodos para estimar o tamanho da parcela experimental é baseada em ensaios de uniformidade (ensaios em branco), nos quais toda a área é plantada com uma única espécie, utilizando-se práticas idênticas de cultivo, sem efeitos de tratamentos (PARANAÍBA et al., 2009). De acordo com Igue et al. (1991), a avaliação da característica de interesse é feita por partes constituídas de dimensões, ou números preestabelecidos, chamadas unidades básicas (UB).

A lei empírica de Smith (1938), que estabeleceu uma relação entre a variância e o tamanho de parcela, foi a principal precursora de vários métodos de determinação do tamanho de parcela (BAKKE, 1988), principalmente pela introdução do conceito do índice de heterogeneidade do solo $b$. Essa relação é descrita por $V_{u x}=V_{l} / X^{b}$, sendo $V_{u x}$ dado por $V_{x} / X^{2}$, com $V_{x}$ igual à variância entre as parcelas de tamanho $X \mathrm{UB}, X$ o tamanho da parcela em UB, $V_{l}$ a variância entre as parcelas compostas de uma UB e $b$ o índice de heterogeneidade do solo. O índice $b$ é uma medida de correlação entre as plantas do experimento e valores próximos à unidade indicam alta heterogeneidade da área ou baixa correlação entre parcelas, o que indica a necessidade de parcelas maiores (IGUE et al., 1991; PARANAÍBA et al., 2009; STORCK et al., 2006).

Cerne, Lavras, v. 17, n. 3, p. 327-338, jul./set. 2011
Um dos métodos mais utilizados na literatura é o da máxima curvatura modificado (SILVA et al., 2003). Foi desenvolvido por Lessman e Atkins (1963) que estabeleceram uma função do tipo $C V_{x}=a / X^{b}$, sendo $C V_{x}$ o coeficiente de variação entre parcelas de tamanho $X$ UB, $X$ o tamanho da parcela em UB e $a$ e $b$ parâmetros a serem estimados. O método permitiu que o ponto que corresponde ao tamanho ótimo da parcela fosse determinado algebricamente, dando maior precisão aos resultados obtidos (VIANA, 1999; VIANA et al., 2002). A partir de então, diversos métodos baseados na máxima curvatura foram desenvolvidos, buscando apurar as estimativas do tamanho ótimo das parcelas.

Um método que também se destaca foi proposto por Hatheway (1961), o qual une a relação empírica de Smith (1938) à fórmula para determinação do número adequado de repetições proposto por Cochran e Cox (1957). Segundo Donato et al. (2008), o método é denominado "tamanho conveniente de parcela" uma vez que estima diferentes tamanhos de parcelas adequados às condições da pesquisa e não apenas um tamanho ótimo.

Dessa forma, definir adequadamente o tamanho das parcelas experimentais para candeia é uma alternativa para garantir interpretações corretas e conclusões bem fundamentadas sobre o cultivo dessa espécie. Assim, com o trabalho, objetivou-se estimar o tamanho ótimo, tamanhos convenientes e forma adequada de parcelas experimentais para Eremanthus erythropappus, para a avaliação das principais características dendrométricas.

\section{MATERIAL E MÉTODOS}

\subsection{Descrição da área de estudo}

A área de estudo localiza-se no município de Aiuruoca, Minas Gerais, nas coordenadas de $21^{\circ} 58^{\prime} 23^{\prime \prime}$ de latitude Sul e $44^{\circ} 44^{\prime} 35^{\prime \prime}$ de longitude Oeste. A altitude varia de 1350 a $1700 \mathrm{~m}$. O clima é mesotérmico úmido do tipo $\mathrm{Cwb}$, tropical de altitude. A temperatura do mês mais quente é inferior a $22^{\circ} \mathrm{C}$, a temperatura média anual é de $18,5^{\circ} \mathrm{C}$ e a média pluviométrica anual é de $1400 \mathrm{~mm}$. $\mathrm{Na}$ região, predomina o Latossolo Vermelho-Amarelo (PÉREZ et al., 2004). A área do ensaio de uniformidade trata-se de um plantio comercial de 5,5 ha de candeia de propriedade da empresa Citróleo Indústria e Comércio de Óleos Essenciais Ltda, instalado no espaçamento $2,5 \times 2,0 \mathrm{~m}$ em janeiro de 2005, sendo que nenhum sistema de manejo ou trato silvicultural foi realizado desde então. 


\subsection{Variáveis dendrométricas analisadas}

Foram mensuradas a circunferência a $1,30 \mathrm{~m}$ de altura do solo $(C A P)$ e altura total $(H T)$ de todos os indivíduos do experimento. O valor da $C A P$ de cada indivíduo com mais de um fuste foi determinado pela fusão dos fustes correspondentes e a $H T$ obtida pela média das alturas desses fustes. O volume total com casca $(V T)$ foi calculado em função das variáveis anteriores, utilizando-se de a equação de volume $\ln (V T)=-10,069537+1,010656 \cdot \ln \left(D A P^{2} \cdot H T\right)$, proposta por Scolforo et al. (2008). Sendo: $V T=$ volume total da árvore com casca $\left(\mathrm{m}^{3}\right) ; D A P=$ diâmetro a $1,30 \mathrm{~m}$ de altura do solo $(\mathrm{cm}) ; H T=$ altura total da árvore $(\mathrm{m})$.

\subsection{Ensaio de uniformidade}

Foram selecionadas dez linhas de plantio, contendo, cada uma, 40 plantas de candeia, formando uma grade de $10 \times 40(i$ linhas $\times j$ planta $)$. Localizada no interior do plantio, evitando-se a borda, de modo a garantir que todas as plantas do ensaio fossem consideradas como plantas úteis. Para o cálculo dos índices, coeficientes e medidas de variação, as plantas foram combinadas de diferentes maneiras, sendo cada planta correspondente a uma UB. A combinação foi feita a partir da soma dos valores observados das UB dentro de cada parcela de tamanho $X$ UB. O processo de combinação para a formação das parcelas e os cálculos foram realizados, utilizando planilhas eletrônicas. $\mathrm{Na}$ Tabela 1, encontram-se todos os 31 agrupamentos possíveis formados pela combinação das 400 UB de modo que linha $\times$ planta $(i \times j)$ forneça somente um número inteiro de parcelas e que se utilize toda a área do ensaio.

\subsection{Métodos utilizados para estimativa do tamanho da parcela}

\subsubsection{Métodos algébricos de curvatura}

Foi utilizado o modelo $C V_{x}=a / X^{b}$ definido por Lessman e Atkins (1963). Sendo que os parâmetros $a$ e $b$ foram estimados, utilizando o software $\mathrm{R}$ ( $\mathrm{R}$ DEVELOPMENT CORE TEAM, 2008). Os pontos de máxima curvatura correspondentes ao tamanho ótimo da parcela foram determinados, utilizando o modelo $X_{0}=\left\{\left[a^{2} b^{2}(2 b+1)\right] /(b+2)\right\}^{[1 /(2 b+2)]}$ definido por Méier e Lessman (1971) e o modelo $X_{0}=\left\{\left[a^{2} b^{2}(2 b-1)\right] /\right.$ $(b-2)\}^{[1 /(2-2 b)]}$ definido por Chaves (1985), codificados como MC 1, MC 2, respectivamente.

Sabendo que $C V_{x}$ se aproxima de um valor assintótico próximo a $180^{\circ}$ quando o tamanho de parcela tende a infinito e partindo da derivação do modelo supracitado, tem-se que $d\left(C V_{x}\right) / d x=-a b / X^{-(b+1)}=\operatorname{tg}(\alpha)$, sendo $175,5^{\circ}$ um valor ótimo de $\alpha$ (OLIVEIRA, 2011). Aplicando-se esses conceitos e isolando $X$, o tamanho estimado resulta no modelo $X_{0}=10^{\log [\operatorname{tg} \alpha)(-a . b)] /(b+1)}$, sendo esse procedimento amplamente utilizado em estudos de redistribuição de água no solo (HILLEL, 1998). Esse método foi codificado como MC 3.

Em relação aos três métodos tem-se: $X_{0}=$ ponto da curvatura correspondente ao tamanho estimado da parcela (UB); $a$ e $b=$ parâmetros estimados do modelo de Lessman e Atkins (1963); $\alpha$ = ângulo dado em radianos, sendo $\alpha_{\text {rad }}=\alpha^{\circ}(\pi / 180)$.

\subsubsection{Modelo linear segmentado com platô}

Para estabelecer uma relação entre o coeficiente de variação e o tamanho da parcela, será utilizado o modelo

Tabela 1 - Combinação das unidades básicas para formação das diferentes parcelas.

Table 1 - Combination of basic units to form different plots.

\begin{tabular}{|c|c|c|c|c|c|c|c|c|c|c|c|c|c|c|c|c|}
\hline \multirow{3}{*}{$i$ Linhas } & \multicolumn{16}{|c|}{$j$ Plantas } \\
\hline & \multicolumn{2}{|c|}{1} & \multicolumn{2}{|c|}{2} & \multicolumn{2}{|c|}{4} & \multicolumn{2}{|c|}{5} & \multicolumn{2}{|c|}{8} & \multicolumn{2}{|c|}{10} & \multicolumn{2}{|c|}{20} & \multicolumn{2}{|c|}{40} \\
\hline & $\mathrm{X}$ & $\mathrm{N}_{\mathrm{x}}$ & $\mathrm{X}$ & $\mathrm{N}_{\mathrm{x}}$ & $\mathrm{X}$ & $\mathrm{N}_{\mathrm{x}}$ & $\mathrm{X}$ & $\mathrm{N}_{\mathrm{x}}$ & $X$ & $\mathrm{~N}_{\mathrm{x}}$ & $\mathrm{X}$ & $\mathrm{N}_{\mathrm{x}}$ & $\mathrm{X}$ & $\mathrm{N}_{\mathrm{x}}$ & $\mathrm{X}$ & $\mathrm{N}_{\mathrm{x}}$ \\
\hline 1 & 1 & 400 & 2 & 200 & 4 & 100 & 5 & 80 & 8 & 50 & 10 & 40 & 20 & 20 & 40 & 10 \\
\hline 2 & 2 & 200 & 4 & 100 & 8 & 50 & 10 & 40 & 16 & 25 & 20 & 20 & 40 & 10 & 80 & 5 \\
\hline 5 & 5 & 80 & 10 & 40 & 20 & 20 & 25 & 16 & 40 & 10 & 50 & 8 & 100 & 4 & 200 & 2 \\
\hline 10 & 10 & 40 & 20 & 20 & 40 & 10 & 50 & 8 & 80 & 5 & 100 & 4 & 200 & 2 & - & - \\
\hline
\end{tabular}

em que: $X=$ tamanho da parcela (UB);

$N_{x}=$ número de parcelas de tamanho $X$ UB cabíveis no ensaio.

Cerne, Lavras, v. 17, n. 3, p. 327-338, jul./set. 2011 
$C V_{x}=a+b X$ para $X \leq X_{0}$ e $C V_{x}=C V_{p}$ para $X \geq X_{0}$, em que: $C V_{x}, X$ e $X_{0}$, já descritos; $C V_{p}=$ coeficiente de variação no ponto correspondente ao platô (\%); $a$ e $b=$ parâmetros a serem estimados. O tamanho ótimo $\left(X_{0}\right)$ corresponde ao ponto no qual o modelo linear se transforma em um platô. O método foi codificado como MLSP. Para ajuste do modelo foi utilizado o software SAEG (UNIVERSIDADE FEDERAL DE VIÇOSA - UFV, 2007).

\subsubsection{Tamanho conveniente (Método de Hatheway)}

O tamanho conveniente das parcelas foi estimado de acordo com o modelo $X_{c}=\left\{\left[2 C V^{2}\left(t_{1}+t_{2}\right)^{2}\right] /\left(r d^{2}\right)\right\}^{1 / b}$, proposto por Hatheway (1961), em que: $X_{c}=$ tamanho conveniente de parcelas (UB); $b=$ índice de heterogeneidade do solo; $d$ = diferença mínima significativa que se deseja detectar entre médias de $I$ tratamentos ( $\%$ da média); $r=$ número de repetições requerido para detectar diferenças de $d \% ; C V_{1}=$ estimativa do coeficiente de variação para parcelas compostas de uma UB (\%); $t_{1}=$ valor tabelado da distribuição $t$ ao nível de significância $\alpha_{1} ; t_{2}=$ valor tabelado da distribuição $t$ correspondente ao nível de significância $\alpha_{2}=2(1-p)$, sendo que $p$ corresponde à probabilidade de se obter resultado significativos. Tendo $t_{1}$ e $t_{2}, g l=(I-1) \times(r-1)$ para um delineamento em blocos inteiramente casualizados.

Foram utilizados níveis de significância $\alpha_{1}=0,05$ e probabilidade $p=0,8$ de encontrar diferenças significativas entre tratamentos $(I)$. As simulações foram feitas com base em: coeficientes de variação $\left(C V_{1}\right)$ correspondentes a cada uma das três variáveis dendrométricas; 3,4 , 5 ou 6 blocos $(r) ; 2$ a 10 tratamentos (I); diferenças entre médias a serem detectadas (d) iguais a $10,15,20 \%$ ou $25 \%$. As simulações e os cálculos foram realizados utilizando planilhas eletrônicas.

$\mathrm{O}$ índice $b$ foi estimado como um coeficiente de regressão linear, pela linearização do modelo de Smith (1938) dado por $\log \left(V_{u x}\right)=\log \left(V_{l}\right)-b \log (X)$ ponderado pelos graus de liberdade associados ao número de parcelas, assim $\left.b=\left\{\Sigma w_{i} x_{i} y_{i}-\left[\left(\Sigma w_{i} x_{i} \cdot \Sigma w_{i} y_{i}\right]\right) / \Sigma w_{i}\right]\right\} /$ $\left\{\Sigma w_{i} x_{i}^{2}-\left[\left(\Sigma w_{i} x_{i}\right)^{2} / \Sigma w_{i}\right]\right\}$, em que: $w_{i}=$ graus de liberdade associado ao número de parcelas $\left(N_{x}-1\right) ; x_{i}=\log (X)$; $y_{i}=\log \left(V_{u x}\right)$.

\subsection{Determinação da forma adequada}

Após determinar o tamanho ótimo da parcela, foram obtidas todas as formas possíveis, de modo que linha $\times$ planta $(i \times j)$ resultassem no tamanho ótimo proposto $\left(i \times j=X_{0}\right)$. Em seguida, calcularam-se os coeficientes de variação $\left(C V_{x}\right)$ e a Informação Relativa $(I R)$ de acordo com Keller (1949), correspondentes a cada forma. Sendo $I R=100\left(V_{1} / V_{c x}\right)$, em que: $I R=$ informação relativa (\%); $V_{l}=$ variância entre parcelas compostas de uma UB; $V_{c x}=$ variância comparável dada por $V_{x} / X$, sendo $V_{x}=$ variância entre parcelas de tamanho $X \mathrm{UB}$ e $X$ o tamanho da parcela (UB). A melhor forma corresponderá à conformação que forneça o menor $C V$ a maior $I R$.

\section{RESULTADOS E DISCUSSÃO}

\subsection{Tamanho estimado da parcela}

Os valores do coeficiente de variação, para diferentes formas e tamanhos de parcelas simuladas estão apresentados na Tabela 2. O coeficiente de variação tende a reduzir com o aumento do número de $\mathrm{UB}$, independente da forma. Essa é uma tendência esperada, uma vez que o aumento de UB tem influência direta no desvio padrão, o qual dá origem ao coeficiente de variação.

Observa-se que a variável $H T$ é a mais uniforme dentre as três, apresentando um coeficiente de variação $\left(C V_{1}\right)$ de $13,9 \%$ seguida da variável $C A P$ com $23,7 \%$ e da variável $V T$ com $54,2 \%$. Os valores máximos de variação encontrados para as variáveis $C A P$ e $H T$ foram respectivamente de 27,9 e $23,2 \%$ para parcelas compostas de duas UB com forma $2 \times 1$. Já para a variável $V T$, o valor máximo de variação foi de $54,2 \%$ e coincidiu com o valor correspondente a parcelas de uma UB. Os valores mínimos de variação foram encontrados para parcelas compostas de 200 UB com forma $5 \times 40$ para as três variáveis $C A P, H T$ e $V T$, sendo seus valores de 2,$5 ; 1,7$ e $8,8 \%$, respectivamente. A mesma tendência das amplitudes de variação em relação às mesmas três variáveis dendrométricas foi observada por Zanon e Stork (2000) para Eucalyptus saligna.

Na Figura 1, encontram-se os ajustes do modelo proposto por Lessman e Atkins (1963) para as três variáveis dendrométricas.

Independente da variável considerada observa-se uma clara tendência exponencial negativa apresentada pelo coeficiente de variação $\left(C V_{x}\right)$ à medida que se aumenta o número de plantas dentro da parcela $(X)$. Esse comportamento é encontrado em todos os trabalhos de determinação de tamanho ótimo de parcelas experimentais e foi descrito primeiramente pela lei empírica de Smith (1938). Observa-se que o modelo proposto por Lessman e Atkins (1963) se ajusta adequadamente a tendência observada nos gráficos, apresentando elevados coeficientes de determinação $\left(R^{2}\right)$ e baixos erros padrões das estimativas $\left(S_{y x}\right)$, justificando seu uso para estimativas de tamanhos de parcelas experimentais.

Cerne, Lavras, v. 17, n. 3, p. 327-338, jul./set. 2011 
Tabela 2 - Coeficiente de variação para as diferentes formas e tamanhos de parcelas.

Table 2 - Coefficient of variation for different shapes and sizes of plots.

\begin{tabular}{|c|c|c|c|c|c|c|c|c|c|}
\hline \multirow{2}{*}{ X (UB) } & \multirow{2}{*}{$\begin{array}{c}\text { Forma } \\
(i \times j)\end{array}$} & \multicolumn{3}{|c|}{$\mathrm{CV}_{\mathrm{x}}(\%)$} & \multirow{2}{*}{ X (UB) } & \multirow{2}{*}{$\begin{array}{c}\text { Forma } \\
(i \times j)\end{array}$} & \multicolumn{3}{|c|}{$\mathrm{CV}_{\mathrm{x}}(\%)$} \\
\hline & & CAP & HT & $\mathrm{VT}$ & & & CAP & $\mathrm{HT}$ & VT \\
\hline 1 & $1 \times 1$ & 23,7 & 13,9 & 54,2 & 20 & $5 \times 4$ & 8,3 & 7,2 & 22 \\
\hline 2 & $1 \times 2$ & 24,8 & 22,4 & 42,4 & 20 & $10 \times 2$ & 7,5 & 7,1 & 18 \\
\hline 2 & $2 \times 1$ & 27,9 & 23,2 & 48,6 & 25 & $5 \times 5$ & 8,7 & 8,2 & 23 \\
\hline 4 & $1 \times 4$ & 17,2 & 15,2 & 33,1 & 40 & $1 \times 40$ & 6,6 & 5,1 & 13 \\
\hline 4 & $2 \times 2$ & 17,8 & 15,8 & 32,5 & 40 & $2 \times 20$ & 7,4 & 5,1 & 17 \\
\hline 5 & $1 \times 5$ & 15,7 & 14,9 & 31,2 & 40 & $5 \times 8$ & 7,6 & 6,8 & 21 \\
\hline 5 & $5 \times 1$ & 15,8 & 13,6 & 32,2 & 40 & $10 \times 4$ & 6,1 & 6,2 & 16 \\
\hline 8 & $1 \times 8$ & 12,5 & 12,1 & 26,4 & 50 & $5 \times 10$ & 7,9 & 7,8 & 18 \\
\hline 8 & $2 \times 4$ & 11,1 & 9,9 & 24,6 & 50 & $10 \times 5$ & 6,1 & 6,2 & 17 \\
\hline 10 & $1 \times 10$ & 12,5 & 11,9 & 23,8 & 80 & $2 \times 40$ & 4,8 & 1,9 & 12 \\
\hline 10 & $2 \times 5$ & 10,7 & 10,2 & 25,7 & 80 & $10 \times 8$ & 5,7 & 6,1 & 15 \\
\hline 10 & $5 \times 2$ & 11,4 & 10,3 & 25,1 & 100 & $5 \times 20$ & 5,8 & 5,2 & 17 \\
\hline 10 & $10 \times 1$ & 11,2 & 9,5 & 23,2 & 100 & $10 \times 10$ & 5,4 & 6,2 & 11 \\
\hline 16 & $2 \times 8$ & 8,9 & 8,1 & 21,8 & 200 & $5 \times 40$ & 2,5 & 1,7 & 8,8 \\
\hline 20 & $1 \times 20$ & 9 & 7,6 & 19 & 200 & $10 \times 20$ & 5,9 & 5,7 & 13 \\
\hline 20 & $2 \times 10$ & 8,6 & 8,2 & 19,1 & - & - & - & - & - \\
\hline
\end{tabular}
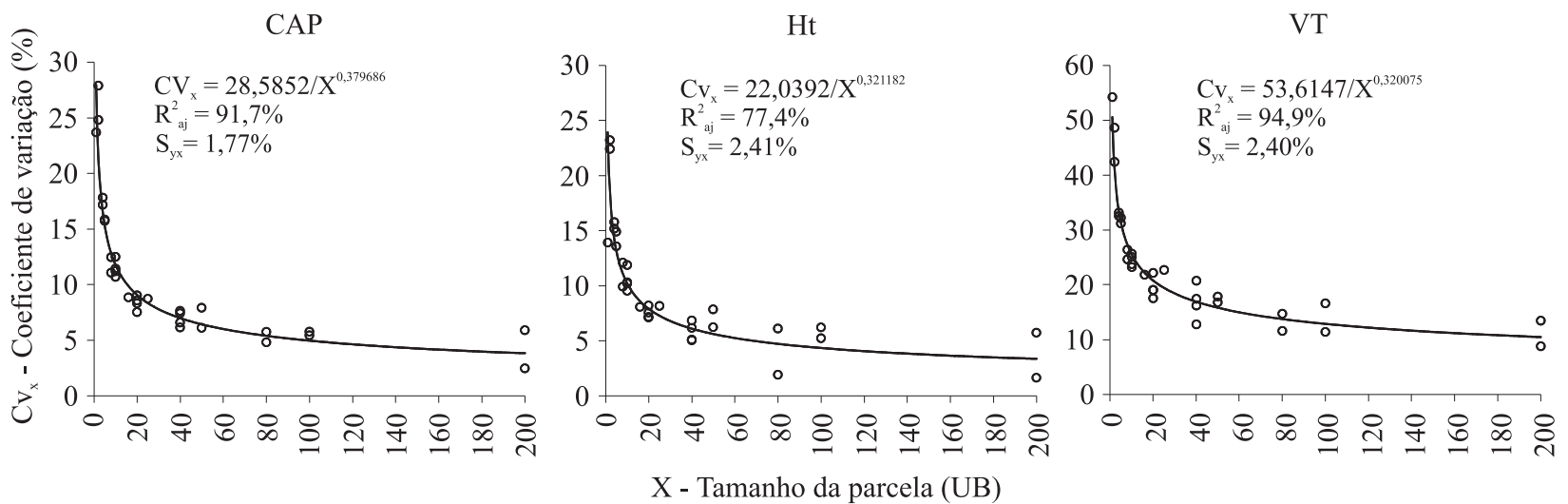

Figura 1 - Ajuste do modelo de Lessman e Atkins (1963) para as variáveis dendrométricas analisadas.

Figure 1 - Fit of the Lessman and Atkins (1963) model, for the analyzed dendrometric variables.

Os ajustes dos modelos segmentados com platô para as três variáveis dendrométricas são apresentados na Figura 2. Os modelos lineares segmentados com platô não se ajustam tão bem quanto o modelo anterior, isso é de se esperar uma vez que tratam-se de dois segmentos de reta para explicar uma tendência exponencial. O mais importante aqui não é o melhor ajuste de modelos, mas sim o ponto de junção das duas retas, indicando o ótimo entre o ganho em precisão e o tamanho da parcela experimental.

Na Tabela 3, encontram-se os tamanhos $\left(X_{0}\right)$ das parcelas experimentais estimados a partir dos diferentes métodos e para as três variáveis.

Cerne, Lavras, v. 17, n. 3, p. 327-338, jul./set. 2011 

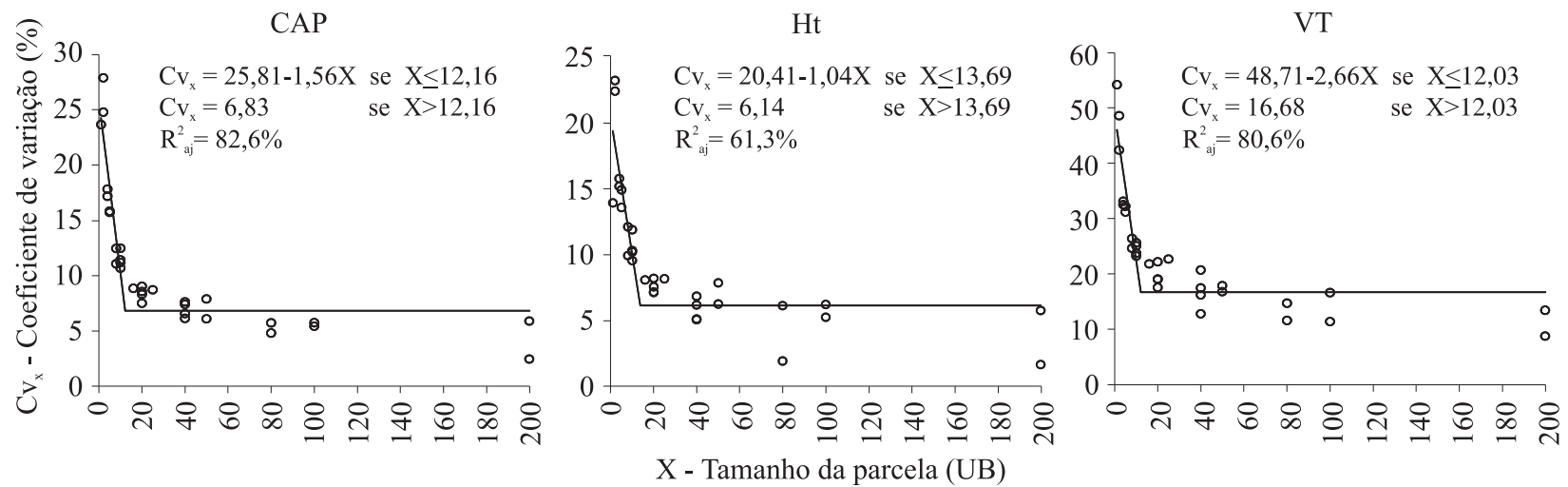

Figura 2 - Ajuste do modelo de linear segmentado com platô para as variáveis dendrométricas analisadas.

Figure 2 - Fit of the segmented linear plateau model, for the analyzed dendrometric variables.

Tabela 3 - Tamanho ótimo de parcelas experimentais para candeia considerando os métodos utilizados e as variáveis dendrométricas analisadas.

Table 3 - Optimal size of experimental plots for candeia, considering the methods used and the analyzed dendrometric variables.

\begin{tabular}{lcccc}
\hline \multirow{2}{*}{ Variável } & \multicolumn{4}{c}{$X_{0}(\mathrm{UB})$} \\
\cline { 2 - 5 }$C A P$ & MC 1 & MC 2 & MC 3 & MLSP \\
$H T$ & 5 & 10 & 36 & 13 \\
$V T$ & 4 & 6 & 31 & 14 \\
\hline
\end{tabular}

Obs.: Os valores foram arredondados para o inteiro superior mais próximo.

Os tamanhos ótimos obtidos pelo método da máxima curvatura a partir do modelo de Meier e Lessman (1971) (MC 1) foram de 4, 5 e 8 UB para as variáveis $H T, C A P$ e $V T$, respectivamente. No entanto, é de convir que os tamanhos de parcelas estimados são inconsistentes e não refletem tamanhos sensatos de parcelas experimentais. Segundo Silva et al. (2003), o método não considera o ponto de estabilização dos valores do coeficiente de variação. Desse modo, o aumento no número de plantas na parcela ainda promove ganho significativo na precisão experimental. Assim, esse método tende a subestimar o número ótimo de plantas por parcela. Feijó et al. (2006), Henriques-Neto et al. (2009) e Lima et al. (2007) também encontraram problemas de subestimação, utilizando esse método.

Chaves (1985) afirmou que os valores encontrados pelo método da máxima curvatura de Méier e Lessman (1971) devem ser interpretados como o limite mínimo de tamanho de parcela e não como tamanho ótimo. Em função disso, propôs um novo modelo para corrigir o problema da subestimação do método anterior. Os tamanhos ótimos obtidos pelo método da máxima curvatura, a partir do modelo de Chaves (1985) (MC 2) foram de 6, 10 e $22 \mathrm{UB}$ para as variáveis $H T, C A P$ e $V T$, respectivamente. Viana et al. (2002, 2003) encontraram tamanhos coerentes com as culturas estudadas, utilizando esse método. Já Vieira e Silva (2008) encontraram problemas de subestimação, porém, esses autores simularam parcelas muito pequenas de no máximo 15 UB. Os tamanhos estimados obtidos pelo método MC 3 foram de 31, 36 e 60 UB para as variáveis $H T, C A P$ e $V T$, respectivamente. Valores esses também coerentes e muito superiores aos estimados pelo método anterior. Nota-se que esse modelo não considera exatamente a máxima curvatura, mas sim um ponto mais próximo à assíntota, em que a redução do coeficiente de variação ainda é considerável. Desse modo, o método estima um tamanho de parcela em que o ganho em precisão é mais significativo, garantindo maior segurança nas avaliações das principais características dendrométricas nos experimentos.

A partir do modelo linear segmentado com platô (MLSP), os tamanhos ótimos estimados foram de 14, $13 \mathrm{e}$ 13 UB para as variáveis $H T, C A P$ e $V T$, respectivamente. Observa-se que os resultados são incoerentes, uma vez que determinaram tamanhos de parcelas semelhantes para as três variáveis dendrométricas, sendo que elas apresentam coeficientes de variação muito distintos. Portanto, este método não apresentou resultados consistentes com a variabilidade dos dados $(\mathrm{CV})$ para esse estudo, diferentemente dos resultados encontrados por Paranaíba et al. (2009), para culturas agrícolas.

Cerne, Lavras, v. 17, n. 3, p. 327-338, jul./set. 2011 
Ambos os método MC 2 e MC 3, apresentaram resultados consistentes para a estimativa do tamanho de parcelas experimentais. Porém, o método MC 3 estima tamanhos em que o ganho em precisão é mais significativo. Portanto, o tamanho adequado da parcela experimental para candeia, de modo a permitir análise das principais características dendrométricas, deve ser de 60 plantas úteis, ou de 36 para análise apenas da CAP e HT.

\subsection{Forma adequada da parcela}

Em relação à forma mais adequada da parcela experimental com 60 plantas, tem-se que esse valor não permite um número inteiro de parcelas e não utiliza toda a área do ensaio. Assim, o número de parcelas de 60 UB foi limitado ao máximo cabível a partir da primeira planta da primeira linha, sendo as conformações possíveis $2 \times 30$, $3 \times 20,4 \times 15$ e $5 \times 12,6 \times 10$ e $10 \times 6$, resultando em cinco, seis, quatro, seis, quatro e seis parcelas para cada uma dessas formas, respectivamente, considerando as $400 \mathrm{UB}$ do ensaio. Na Tabela 4, são apresentadas as comparações entre elas para as três variáveis dendrométricas.
As formas mais adequadas para a análise das características $C A P, H T$ e $V T$ foram respectivamente, $4 \times 15,4 \times 15$ e $2 \times 30$. Essas formas apresentaram menores coeficientes de variação $(C V)$ e maiores informações relativas $(I R)$. Portanto, o acréscimo de plantas dentro da linha favorece o ganho em precisão para as três variáveis dendrométricas, porém, para a CAP e $H T$, isso ocorre até certo limite. Cocco et al. (2009) e Lúcio et al. (2004), estudando outras culturas observaram que o aumento da parcela no sentido das linhas promovia maior ganho em precisão. Assim como Mello et al. (2009), analisando a estimativa de volume em plantios de Eucalyptus grandis concluíram que, para uma baixa intensidade amostral, parcelas de inventário em linhas garantiam maior exatidão nas estimativas.

No entanto, é impraticável a adoção de formas diferentes para cada variável de interesse em um experimento. É necessário chegar a uma forma que atenda, adequadamente, a todas as variáveis. A princípio, seria lógica a opção pela forma $4 \times 15$, por atender melhor à $C A P$ e à $H T$, no entanto, $V T$ é a variável mais crítica em

Tabela 4 - Análise da forma adequada de parcelas compostas de 60 plantas para as variáveis dendrométricas.

Table 4 - Analysis of suitable shape of plots comprising 60 plants, for the analyzed dendrometric variables.

\begin{tabular}{|c|c|c|c|c|c|c|}
\hline Variável & Forma $(i \times j)$ & $V_{1}$ & $V_{x}$ & $V_{c x}$ & $C V_{60}(\%)$ & $I R(\%)$ \\
\hline \multirow{6}{*}{$C A P$} & $2 \times 30$ & 19,09 & 1830,97 & 30,52 & 4,5 & 62,6 \\
\hline & $3 \times 20$ & 18,07 & 2531,32 & 42,19 & 5,1 & 42,8 \\
\hline & $4 \times 15$ & 20,80 & 1173,87 & 19,56 & 3,5 & 106,3 \\
\hline & $5 \times 12$ & 18,47 & 3824,12 & 63,74 & 6,4 & 29,0 \\
\hline & $6 \times 10$ & 20,44 & 5843,52 & 97,39 & 7,6 & 21,0 \\
\hline & $10 \times 6$ & 18,47 & 3948,38 & 65,81 & 6,5 & 28,1 \\
\hline \multirow{6}{*}{$H T$} & $2 \times 30$ & 0,30 & 29,40 & 0,49 & 2,6 & 61,1 \\
\hline & $3 \times 20$ & 0,30 & 90,67 & 1,51 & 4,5 & 19,6 \\
\hline & $4 \times 15$ & 0,31 & 25,80 & 0,43 & 2,5 & 72,5 \\
\hline & $5 \times 12$ & 0,29 & 125,37 & 2,09 & 5,3 & 13,9 \\
\hline & $6 \times 10$ & 0,30 & 272,81 & 4,55 & 7,8 & 6,6 \\
\hline & $10 \times 6$ & 0,29 & 153,03 & 2,55 & 5,9 & 11,4 \\
\hline \multirow{6}{*}{$V T$} & $2 \times 30$ & $1,11.10^{-5}$ & $9,27 \cdot 10^{-4}$ & $1,55.10^{-5}$ & 9,7 & 72,1 \\
\hline & $3 \times 20$ & $1,07.10^{-5}$ & $2,43 \cdot 10^{-3}$ & $4,06.10^{-5}$ & 14,7 & 26,3 \\
\hline & $4 \times 15$ & $1,24.10^{-5}$ & $2,52.10^{-3}$ & $4,20.10^{-5}$ & 15,4 & 29,6 \\
\hline & $5 \times 12$ & $1,07.10^{-5}$ & $4,27 \cdot 10^{-3}$ & $7,12 \cdot 10^{-5}$ & 20,2 & 15,0 \\
\hline & $6 \times 10$ & $1,26.10^{-5}$ & $4,17.10^{-3}$ & $6,95 \cdot 10^{-5}$ & 18,6 & 18,1 \\
\hline & $10 \times 6$ & $1,07.10^{-5}$ & $2,93 \cdot 10^{-3}$ & $4,88.10^{-5}$ & 16,7 & 21,9 \\
\hline
\end{tabular}

Em que: $V_{l}=$ variância entre as parcelas compostas de uma UB; $V_{x}=$ variância entre as parcelas de tamanho $X$ UB; $V_{c x}=$ variância comparável dada por $V_{x} / X$.

Cerne, Lavras, v. 17, n. 3, p. 327-338, jul./set. 2011 
termos de variabilidade. A adoção da forma $4 \times 15$ levaria a uma perda considerável de precisão nas análises de $V T$, aumentando o $C V$ de 9,7 para $15,4 \%$ e redução da $I R$ de 72,1 para $29,6 \%$. Já a conformação $2 \times 30$ se torna uma opção sensata, uma vez que representa a forma mais adequada para $V T$ e a segunda melhor forma para $C A P \mathrm{e}$ $H T$, sendo que acarreta poucas perdas em precisão para essas duas últimas. Portanto, a forma mais adequada de parcelas experimentais para candeia, de modo a permitir análise das principais características dendrométricas deve ser $2 \times 30$, ou seja, duas linhas com 30 plantas.

\subsection{Tamanho conveniente da parcela}

Os ajustes para estimativas dos coeficientes de heterogeneidade do solo $b$ para as três variáveis dendrométricas são apresentados na Figura 3.

Os coeficientes de heterogeneidade encontrados foram de 0,$773 ; 0,539$ e 0,716 para $C A P, H T$ e $V T$, respectivamente. Valores de $b$ para $C A P$ e $V T$ indicam alta heterogeneidade do local e baixa correlação entre as unidades básicas, o que implica na necessidade de parcelas maiores para essas variáveis. Lin e Binns (1986) afirmaram que no caso de $b$ maior que 0,7 um aumento no tamanho da parcela é mais eficaz para melhorar a precisão experimental do que o aumento no número de repetições. Enquanto no caso da variável $H T, b$ está entre 0,2 e 0,7 indicando que mudanças no tamanho da parcela e no número de repetições podem ser usadas em combinação para aumentar a precisão experimental.

$\mathrm{Na}$ Tabela 5, são apresentados os tamanhos convenientes de parcelas experimentais para candeia, estimados a partir do método de Hatheway (1961), de acordo com as características do experimento e da precisão desejada.
A relação entre o tamanho da parcela e as variáveis do método de Hatheway (1961) é claramente observada. Tem-se que a redução do tamanho conveniente da parcela experimental $\left(X_{c}\right)$ é proporcionada pelo aumento do número de repetições $(r)$, aumento do número de tratamentos $(I)$, aumento da diferença a ser detectada entre tratamentos $(d)$ e pela redução do coeficiente de variação (CV). Muniz et al. (1999, 2009), Simplício et al. (1996) e Viana et al. (2003) descreveram as mesmas relações independente das culturas estudadas.

$\mathrm{O}$ aumento do coeficiente de variação $(\mathrm{CV})$ dentre as variáveis dendrométricas, proporciona aumentos expressivos no tamanho da parcela, chegando a valores impraticáveis. Assim como o aumento do rigor e da precisão exigidos como reflexo da redução da diferença a ser detectada entre tratamentos $(d)$ também proporciona aumentos drásticos na parcela. Já o aumento do número de repetições influencia menos e o de tratamentos ainda menos. Portanto, os efeitos mais significativos sobre o tamanho da parcela obedecem a ordem $C V>d>r>I$. Sendo, principalmente, a relação $(C V / d)^{2}$ observada por Oliveira (1976) a mais importante.

Em experimentos florestais não é comum a análise somente da variável $H T$, certamente a $C A P$ será analisada conjuntamente e, eventualmente, o VT. Assim, a discussão será limitada ao $C A P$ e $V T$, sendo que os tamanhos convenientes para essas duas serão seguramente suficientes para análises de $H T$.

No caso da variável $C A P$, é perfeitamente possível a conciliação entre precisão e tamanho da parcelas. É possível detectar pequenas diferenças de $10 \%(d)$ entre tratamentos utilizando quatro blocos com seis a dez tratamentos, resultando em parcelas a 61 a 66 plantas. $\mathrm{Ou}$ ainda utilizando cinco blocos com três a dez tratamentos

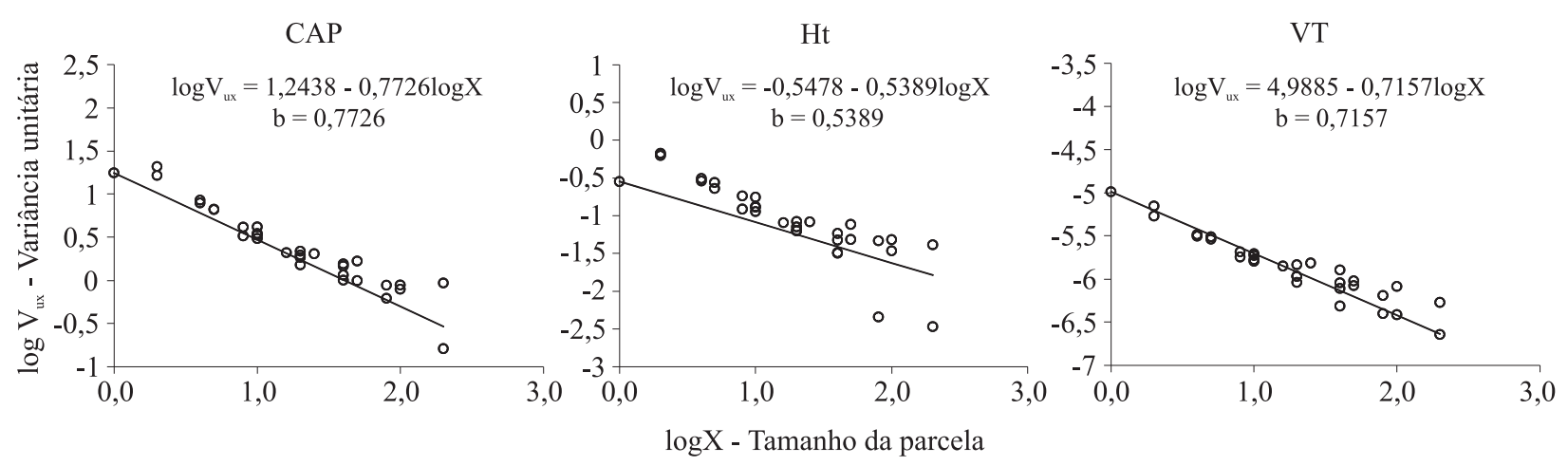

Figura 3 - Estimativa do coeficiente de heterogeneidade $b$ para as variáveis dendrométricas analisadas.

Figure 3 - Estimate of the heterogeneity index b for the analyzed dendrometric variables.

Cerne, Lavras, v. 17, n. 3, p. 327-338, jul./set. 2011 
Tabela 5 - Tamanhos convenientes de parcelas experimentais para candeia para as variáveis dendrométricas analisadas.

Table 5 - Convenient sizes for experimental plots of candeia, for the analyzed dendrometric variables.

\begin{tabular}{|c|c|c|c|c|c|c|c|c|c|c|c|}
\hline \multirow{3}{*}{ Variável } & \multirow{3}{*}{$\begin{array}{c}\text { DMS } \\
\text { (d) }\end{array}$} & \multirow{3}{*}{$\begin{array}{c}\text { Blocos } \\
\text { (r) }\end{array}$} & \multicolumn{9}{|c|}{$\mathrm{X}_{\mathrm{c}}(\mathrm{UB})$} \\
\hline & & & \multicolumn{9}{|c|}{ Tratamentos (I) } \\
\hline & & & 2 & 3 & 4 & 5 & 6 & 7 & 8 & 9 & 10 \\
\hline \multirow{9}{*}{$\begin{array}{l}\text { CAP } \\
C V=23,3 \% \\
b=0,772\end{array}$} & $10 \%$ & 3 & 426 & 165 & 127 & 112 & 104 & 99 & 96 & 94 & 92 \\
\hline & $15 \%$ & 3 & 150 & 58 & 45 & 39 & 37 & 35 & 34 & 33 & 33 \\
\hline & $20 \%$ & 3 & 71 & 28 & 21 & 19 & 18 & 17 & 16 & 16 & 16 \\
\hline & $10 \%$ & 4 & 153 & 87 & 74 & 69 & 66 & 64 & 62 & 61 & 61 \\
\hline & $15 \%$ & 4 & 54 & 31 & 26 & 24 & 23 & 23 & 22 & 22 & 22 \\
\hline & $20 \%$ & 4 & 26 & 15 & 13 & 12 & 11 & 11 & 11 & 11 & 10 \\
\hline & $10 \%$ & 5 & 86 & 58 & 52 & 49 & 47 & 46 & 45 & 45 & 44 \\
\hline & $15 \%$ & 5 & 30 & 21 & 18 & 17 & 17 & 16 & 16 & 16 & 16 \\
\hline & $20 \%$ & 5 & 15 & 10 & 9 & 9 & 8 & 8 & 8 & 8 & 8 \\
\hline \multirow{9}{*}{$\begin{array}{l}\text { HT } \\
\text { CV }=13,9 \% \\
b=0,539\end{array}$} & $10 \%$ & 3 & 820 & 211 & 144 & 120 & 109 & 102 & 97 & 94 & 91 \\
\hline & $15 \%$ & 3 & 183 & 47 & 32 & 27 & 25 & 23 & 22 & 21 & 21 \\
\hline & $20 \%$ & 3 & 63 & 17 & 11 & 10 & 9 & 8 & 8 & 8 & 7 \\
\hline & $10 \%$ & 4 & 188 & 85 & 67 & 60 & 56 & 54 & 52 & 51 & 50 \\
\hline & $15 \%$ & 4 & 42 & 19 & 15 & 14 & 13 & 12 & 12 & 12 & 11 \\
\hline & $20 \%$ & 4 & 15 & 7 & 6 & 5 & 5 & 5 & 4 & 4 & 4 \\
\hline & $10 \%$ & 5 & 82 & 47 & 40 & 37 & 35 & 34 & 33 & 33 & 32 \\
\hline & $15 \%$ & 5 & 19 & 11 & 9 & 9 & 8 & 8 & 8 & 8 & 8 \\
\hline & $20 \%$ & 5 & 7 & 4 & 3 & 3 & 3 & 3 & 3 & 3 & 3 \\
\hline \multirow{9}{*}{$\begin{array}{l}\mathrm{VT} \\
\mathrm{CV}=54,2 \% \\
\mathrm{~b}=0,716\end{array}$} & $15 \%$ & 4 & 740 & 405 & 339 & 311 & 296 & 287 & 280 & 276 & 272 \\
\hline & $20 \%$ & 4 & 331 & 182 & 152 & 140 & 133 & 129 & 126 & 124 & 122 \\
\hline & $25 \%$ & 4 & 178 & 98 & 82 & 75 & 71 & 69 & 68 & 66 & 66 \\
\hline & $15 \%$ & 5 & 396 & 259 & 228 & 214 & 207 & 202 & 199 & 196 & 194 \\
\hline & $20 \%$ & 5 & 177 & 116 & 102 & 96 & 93 & 91 & 89 & 88 & 87 \\
\hline & $25 \%$ & 5 & 95 & 63 & 55 & 52 & 50 & 49 & 48 & 47 & 47 \\
\hline & $15 \%$ & 6 & 257 & 186 & 168 & 160 & 156 & 153 & 151 & 149 & 148 \\
\hline & $20 \%$ & 6 & 115 & 84 & 76 & 72 & 70 & 69 & 68 & 67 & 67 \\
\hline & $25 \%$ & 6 & 62 & 45 & 41 & 39 & 38 & 37 & 37 & 36 & 36 \\
\hline
\end{tabular}

Em que: $X_{c}=$ tamanho conveniente da parcela (UB); $D M S=$ diferença mínima significativa a ser detectada entre médias tratamentos (\%).

resultando em parcelas de 44 a 58 plantas. Com uma precisão menos rigorosa com $d$ de $15 \%$ é possível utilizar até três blocos, desde que se utilizem três ou mais tratamentos, ou utilizar quatro ou cinco blocos independente do número de tratamentos, resultando em parcelas de 16 a 58 plantas. Assim, não é sensato sacrificar a precisão para uma $d$ maior ou igual $20 \%$ já que é possível obter parcelas praticáveis com maior precisão ( $d$ igual a 10 ou $15 \%$ ).
No caso da variável $V T$, em consequência da sua alta variabilidade, poucas situações descritas na Tabela 5 denotam tamanhos de parcelas praticáveis. Utilizando quatro blocos com seis a dez tratamentos, ou cinco blocos com três a dez tratamentos, ou seis blocos independente do número de tratamentos, seria possível ter parcelas de 36 a 71 plantas. No entanto, teria-se precisão mais baixa, sendo possível detectar apenas diferenças entre médias de

Cerne, Lavras, v. 17, n. 3, p. 327-338, jul./set. 2011 
tratamentos se elas divergissem em $25 \%$ (d). Diferenças de $20 \%$ seriam possíveis de serem detectadas para parcelas de 67 a 70 plantas, se fossem utilizados seis blocos com seis a dez tratamentos, porém, possivelmente, limitado em termos de área experimental. No caso de diferenças menores que $15 \%(d)$, todos os tamanhos de parcelas apresentados são praticamente inviáveis.

Para a análise adequada de experimentos em termos de volume total $(V T)$ necessariamente serão necessárias áreas experimentais muito grandes ou haverá perda de precisão. Portanto, uma estratégia razoável para a análise de experimentos com candeia é utilizar a variável circunferência ou diâmetro $(C A P$ ou $D A P)$ sempre que for o caso, para a interpretação das respostas dos tratamentos. Uma vez que é possível obter alta precisão com tamanhos de parcelas praticáveis e ainda os tamanhos adotados contemplarão respostas em altura (HT).

\section{CONCLUSÕES}

O tamanho de parcelas experimentais para candeia, de modo a permitir análise das principais características dendrométricas, deve ser de 60 plantas úteis.

A conformação retangular $2 \times 30$ (linhas $\times$ plantas) é a forma mais adequada para as parcelas experimentais para candeia, devendo ser alocada perpendicularmente a possíveis fatores do terreno que causem heterogeneidade.

O tamanho conveniente de parcela estimado pelo método de Hatheway é muito flexível e permite adequação do tamanho da parcela experimental com as condições, características e limitações do experimento.

Em experimentos com candeia deve-se utilizar a variável circunferência ou diâmetro para a análise das respostas dos tratamentos, uma vez que é possível obter alta precisão com tamanhos de parcelas e áreas experimentais viáveis.

\section{REFERÊNCIAS}

BAKKE, O. A. Tamanho e forma ótimos de parcelas em delineamentos experimentais. 1988. 142 f. Dissertação (Mestrado em Estatística e Experimentação Agronômica) Escola Superior de Agricultura "Luiz de Queiroz", Piracicaba, 1988.

CHAVES, L. J. Tamanho da parcela para seleção de progênies de milho (Zea mays L.). 1985. 148 f. Tese (Doutorado em Estatística e Experimentação Agronômica) -
Escola Superior de Agricultura "Luiz de Queiroz", Piracicaba, 1985.

COCCO, C. et al. Tamanho e forma de parcela em experimentos com morangueiro cultivado em solo ou em hidroponia. Pesquisa Agropecuária Brasileira, Brasília, v. 44, n. 7, p. 681-686, jul. 2009.

COCHRAN, E.; COX, G. M. Experimental designs. 2. ed. New York, J. Wiley, 1957. 611 p.

DONATO, S. L. R. et al. Estimativas de tamanho de parcelas para avaliação de descritores fenotípicos em bananeira.

Pesquisa Agropecuária Brasileira, Brasília, v. 43, n. 8, p. 957-969, ago. 2008.

FEIJÓ, S. et al. Heterogeneidade do solo e de tamanho de amostra antes e após cultivos com abobrinha italiana em estufa plástica. Ciência Rural, Santa Maria, v. 36, n. 6, p. 17441748, nov./dez. 2006.

HATHEWAY, W. H. Convenient plot size. Agronomy Journal, Madison, v. 53, n. 4, p. 279-280, 1961.

HENRIQUES-NETO, D. et al. Tamanho de parcela para avaliação da produção em trigo irrigado, sob dois sistemas de plantio. Ciência Agronômica, Jaboticabal, v. 40, n. 1, p. 8693, jan./mar. 2009.

HILLEL, D. Environmental soil physics. San Diego: Academic, 1998. 767 p.

IGUE, T. et al. Tamanho e forma de parcela experimental para cana-de-açúcar. Bragantia, Campinas, v. 50, n. 1, p. 163-180, 1991.

KELLER, K. R. Uniformity trial on hops, Humulus lupulus L., for increasing the precision of field experiments. Agronomy Journal, Madison, v. 41, n. 8, p. 389-392, 1949.

LESSMAN, K. J.; ATKINS, R. E. Optimum plot size and relative efficiency flattice designs for grain sorghum yield tests. Crop Science, Madison, v. 3, n. 5, p. 477-481, 1963.

LIMA, J. F. et al. Tamanho ótimo de parcela para experimentos com plantas de mamoeiro em casa de vegetação. Ciência e Agrotecnologia, Lavras, v. 31, n. 5, p. 1411-1415, set./out. 2007.

Cerne, Lavras, v. 17, n. 3, p. 327-338, jul./set. 2011 
LIN, C. S.; BINNS, M. R. Relative efficiency of two randomized block designs having different plot sizes and numbers of replications and of plots per block. Agronomy Journal, Madison, v. 78, n. 3, p. 531-534, 1986.

LÚCIO, A. D. et al. Estimativa de parâmetros para o planejamento de experimentos com a cultura do pimentão em área restrita. Horticultura Brasileira, Brasília, v. 22, n. 4, p. 766-770, 2004.

MEIER, V. D.; LESSMAN, K. J. Estimation of optimum field plot shape and size for testing yield in Crambe abyssinica Hochst. Crop Science, Madison, v. 11, n. 5, p. 648-650, 1971.

MELLO, J. M. et al. Métodos de amostragem e geoestatística para estimativa do número de fustes e volume em plantios de Eucalyptus grandis. Floresta, Curitiba, v. 39, n. 1, p. 157-166, 2009.

MUNIZ, J. A. et al. Determinação do tamanho de parcelas experimentais em povoamentos de Eucalyptus grandis Mill.: II., parcelas quadradas. Ciência e Agrotecnologia, Lavras, v. 23, n. 2, p. 446-453, mar./abr. 1999.

MUNIZ, J. A. et al. Estudo do tamanho de parcelas experimentais em povoamentos de Eucalyptus grandis hill, usando parcelas lineares. Ciência e Agrotecnologia, Lavras, v. 33, n. 4, p. 1002-1010, jul./ago. 2009.

OLIVEIRA, A. D. et al. Market chain analysis of candeia timber (Eremanthus erythropappus). Cerne, Lavras, v. 15, n. 3, p. 257-264, jul./set. 2009.

OLIVEIRA, G. M. V. Tamanho de parcelas pxperimentais para Eremanthus erythropappus. 2011. 119 p. Dissertação (Mestrado em Engenharia Florestal) - Universidade Federal de Lavras, Lavras, 2011.

OLIVEIRA, R. P. de. Estudo comparativo de alguns métodos de estimação do tamanho adequado de parcelas experimentais. 1976. 110 f. Dissertação (Mestrado) Universidade de Brasília, Brasília, 1976.

PARANAÍBA, P. F.; FERREIRA, D. F.; MORAIS, A. R. Tamanho ótimo de parcelas experimentais, proposição de métodos de estimação. Revista Brasileira de Biometria, São Paulo, v. 27, n. 2, p. 255-268, 2009.
PÉREZ, J. F. M. et al. Sistema de manejo para a candeia Eremanthus erythropappus (dc.) macleish: a opção do sistema de corte seletivo. Cerne, Lavras, v. 10, n. 2, p. 257-273, jul./ dez. 2004.

R DEVELOPMENT CORE TEAM. R: a language and environment for statistical computing. Vienna: R Foundation for Statistical Computing, 2008. Disponível em: <http:// www.R-project.org>. Acesso em: 10 jun. 2010.

SCOLFORO, J. R. S.; OLIVEIRA, A. D. de; ACERBI JUNIOR, F. W. Inventário florestal de Minas Gerais: equações de volume, peso de matéria seca e carbono para diferentes fisionomias da flora nativa. Lavras: UFLA, 2008. 216 p.

SILVA, C. P. de C. Crescimento e produção da candeia em plantio sujeito a diferentes espaçamentos e podas. 2009. 136 p. Dissertação (Mestrado em Engenharia Florestal) Universidade Federal de Lavras, Lavras, 2009.

SILVA, R. L. et al. Determinação do tamanho ótimo da parcela experimental pelos métodos da máxima curvatura modificado, do coeficiente de correlação intraclasse e da análise visual em testes clonais de eucalipto. Revista Árvore, Viçosa, v. 27, n. 5 , p. $669-676,2003$.

SIMPLÍCIO, E. et al. Determinação do tamanho de parcelas experimentais em povoamentos de Eucalyptus grandis Hill ex-Maiden: I., parcelas retangulares. Cerne, Lavras, v. 2, n. 1, p. 53-65, jan./jun. 1996.

SMITH, H. F. An empirical law describing heterogeneity in the yields of agricultural crops. Journal of Agricultural Science, Cambridge, v. 28, p. 1-23, 1938.

STORCK, L. et al. Tamanho ótimo de parcela em experimentos com milho relacionado a metodologias. Revista Brasileira de Milho e Sorgo, Sete Lagoas, v. 5, n. 1, p. 48-57, 2006.

\section{UNIVERSIDADE FEDERAL DE VIÇOSA. Sistema para} análises estatísticas, versão 9.1 demo. Viçosa, MG: Fundação Arthur Bernardes, 2007.

VIANA, A. E. S. Estimativas do tamanho de parcela e característica do material de plantio em experimentos com Manihot esculenta Crantz. 1999. 132 p. Tese (Doutorado em Genética e Melhoramento) - Universidade Federal de Viçosa, Viçosa, 1999.

Cerne, Lavras, v. 17, n. 3, p. 327-338, jul./set. 2011 
VIANA, A. E. S. et al. Estimativas de tamanho de parcela em experimentos com mandioca. Horticultura Brasileira, Brasília, v. 20, n. 1, p. 58-63, mar. 2002.

VIANA, A. E. S. et al. Estudos sobre tamanho de parcela em experimentos com mandioca (Manihot esculenta Crantz).

Acta Scientiarum Agronomy, Maringá, v. 25, n. 2, p. 281289, 2003.
VIEIRA, J. V.; SILVA, G. O. Tamanho mínimo de parcela para avaliação de caracteres de raiz em cenoura. Bragantia, Campinas, v. 67, n. 4, p. 1047-1052, 2008.

ZANON, M. L. B.; STORCK, L. Tamanho ótimo de parcelas experimentais para Eucalyptus saligna Smith em dois estádios de desenvolvimento. Cerne, Lavras, v. 6, n. 2, p. 104-111, jul./ dez. 2000.

Cerne, Lavras, v. 17, n. 3, p. 327-338, jul./set. 2011 